\title{
Poor physical fitness is associated with impaired memory, executive function, and depression in institutionalized older adults: a cross-sectional study
}

\author{
Renato S. Monteiro-Junior, ${ }^{1}$ (iD Tayrine R. Oliveira, ${ }^{2}$ Luana L. Leão, ${ }^{1}$ iD Marcelo P. Baldo, ${ }^{1}$ \\ Alfredo M. de Paula, ${ }^{1}$ Jerson Laks ${ }^{3}$ \\ ${ }^{1}$ Programa de Pós-Graduação em Ciências da Saúde, Universidade Estadual de Montes Claros, Montes Claros, MG, Brazil. ${ }^{2}$ Instituto de \\ Neurociência do Exercício, Hospital Aroldo Tourinho, Montes Claros, MG, Brazil. ${ }^{3}$ Instituto de Psiquiatria, Universidade Federal do Rio de \\ Janeiro, Rio de Janeiro, RJ, Brazil.
}

\begin{abstract}
Objective: To analyze the association between physical fitness, cognitive function, and depressive symptoms among older adults in long-term care facilities (LTCFs).

Methods: Seventy-six institutionalized male and female elderly individuals (65 years and older) living in LTCFs participated of this study. Physical fitness (aerobic capacity and strength), cognitive functions (global cognition, short-term and working and semantic memories, and executive function), and depressive symptoms were assessed. Linear regression and contingency analyses were performed. Significance was accepted at $p$-values $\leqslant 0.05$.

Results: Aerobic capacity predicted $32 \%$ of variance in global cognition $(p<0.01$ ) and $25 \%$ of variance in semantic fluency/executive function $(p<0.01)$. Low levels of upper limb strength, lower limb strength, and aerobic capacity were associated with semantic fluency/executive function $(O R=$ 1.38, $p=0.01$, OR $=1.26, p=0.03$, and $O R=1.07, p=0.01$, respectively) and depressive symptoms $(\mathrm{OR}=1.06, \mathrm{p}<0.01)$.

Conclusion: Poor physical fitness is associated with cognition and depressive symptoms in institutionalized older adults. Low levels of strength and aerobic fitness increase the odds of presenting with impaired semantic fluency and executive function, possibly denoting an increased risk of developing dementia.
\end{abstract}

Keywords: Older adults; nursing home; physical activity; cognition

\section{Introduction}

With worldwide improvements in life expectancy, it is expected that more than $15 \%$ of the global population will be composed of individuals over age 65 by 2050 , while children under age 5 will represent less than $8 \%$ of the total population. ${ }^{1}$ As a result of this increase in life expectancy, however, several noncommunicable diseases related to unsuccessful aging are on the rise, causing concern among international organizations.

Aging is a physiological process, but unsuccessful aging increases the risk of chronic diseases and disabilities. ${ }^{1}$ Loss of function is one of the main reasons older adults are institutionalized in long-term care facilities (LTCFs). Factors that have been related to decreased physical function and frailty include sarcopenia and cognitive decline..$^{2,3}$ Institutionalized and frail elderly have poor physical function and are at high risk of developing cognitive disorders. ${ }^{4,5}$ As these people have few physical

Correspondence: Renato S. Monteiro-Junior, Universidade Estadual de Montes Claros, Av. Doutor Ruy Braga, s $/ n^{\circ}$, Vila Mauriceia, CEP 39401-089, Montes Claros, MG, Brazil.

E-mail: renato.monteiro@unimontes.br

Submitted Nov 03 2020, accepted Jan 07 2021, Epub Apr 162021. and cognitive challenges due to an impoverished environment (e.g., they do not use public transport, handle money, or cook their own meals), the disuse cycle may worsen their functionality and trigger depressive symptoms. ${ }^{6,7}$

Physical fitness is closely related to brain health. The more fit a person is, the better their brain functioning. ${ }^{8}$ Cognition and mood are directly and positively affected by physical capabilities. ${ }^{7,9}$ Plácido et al. ${ }^{10}$ recently found an association among cardiorespiratory fitness, functional status, and dementia. According to this study, older adults with poor aerobic resistance (below normative scores for age) have a 10- to 14-fold higher likelihood of developing mild and moderate dementia, respectively. In addition, these authors showed that cardiorespiratory fitness scores were related to global cognition scores. Regarding depressive symptoms, there is substantial evidence showing a relationship between physical activity level and depression. ${ }^{11,12}$

How to cite this article: Monteiro-Junior RS, Oliveira TR, Leão LL, Baldo MP, de Paula AM, Laks J. Poor physical fitness is associated with impaired memory, executive function, and depression in institutionalized older adults: a cross-sectional study. Braz J Psychiatry. 2022;44:41-45. http://dx.doi.org/10.1590/1516-4446-20201614 
As institutionalized elderly are frequently frail and have both cognitive impairments and depressive symptoms, ${ }^{3,6,13}$ the relationship between physical fitness and mental health seems to be more relevant to this population. Indeed, studies show that exercises can effectively improve everyday functionality, mobility, and have positive effects on cognitive performance of institutionalized older adults. ${ }^{14,15}$ Therefore, if an association between physical capabilities and mental health is found for individuals in LTCFs, this would inform managers and health care providers on the need to incorporate physical exercise into routine care at these facilities to improve the general health of their residents. Furthermore, understanding how physical fitness, specific cognitive functions (e.g., short-term and working memory), and depressive symptoms are related could help us to plan specific strategies to improve mental health. The aim of this study was to examine the association among physical fitness, cognitive functions, and depressive symptoms in older adults living in LTCFs.

\section{Methods}

\section{Participants}

Seventy-six male and female elderly individuals (65 years and above) living in LTCFs were recruited. They met the following inclusion/exclusion criteria: i) independent ambulation; ii) preserved communication skills and comprehension of commands (assessed through an interview); iii) no severe cardiovascular disease; iv) no acute musculoskeletal injury; v) no delirium. These criteria were applied by a health professional. All individuals were screened for dementia by a physician. Participants with a reliable diagnosis of dementia were excluded, while individuals with probable dementia remained in the sample. Symptoms of depression were assessed between men and women for homogeneity purposes.

Sociodemographic variables of each participant such as age, comorbidities, duration of LTCF residence, and medications were retrieved from records in each LTCF. Data were collected from five separate LTCFs in two Brazilian cities (Rio de Janeiro and Montes Claros).

\section{Cognitive assessments}

Global cognition was examined using the Portuguese version of the Mini-Mental State Examination (MMSE). ${ }^{16}$ The following cutoff points were used to determine impairment, while correcting for educational attainment: 13 if illiterate, 18 if less than 9 years of education, and 26 if 9 years of education or more. ${ }^{16}$ Years of education were retrieved from the records in each LTCF if available.

Short-term and working memories were evaluated through Digit Span forward and backward (DSF and DSB) tasks, respectively. The DSF is composed of seven pairs of number sequences. An assessor read each pair of sequences out loud to the participant and asked them to repeat the sequence back in the exact order it was read. When the participant was not able to repeat a pair of sequences correctly, the test was terminated. Each correct response is equivalent to one point, resulting in a total possible score of 14 points. DSB is applied in the same manner as DSF. However, the number sequences should be repeated by the participant in reverse order. ${ }^{17}$ Cutoff scores for DSF and DSB are 6 and 4, respectively, according to median values for people above $60 .^{16,18}$

To evaluate semantic fluency and executive function, a verbal fluency (VF, animal category) test was applied. ${ }^{19,20}$ Participants were asked to name as many animals as possible in 1 minute. A score of 13 was used as a cutoff point to determine low or high executive function, based on a previously published study. ${ }^{19}$ Although that study showed an influence of years of education (illiterate = 11.9 ; up 4 years of education $=12.8$; 4 to 7 years $=13.4$; and 8 years or more $=15.8$ ), we decided to determine 13 points as the cutoff because illiterate persons and those with low or intermediate education showed similar results. Moreover, having more than 8 years of schooling is unusual among residents of the study LTCFs.

All cognitive assessments were conducted in a single day in the afternoon.

\section{Depressive symptoms}

The Brazilian version of the Geriatric Depression Scale (GDS) was used to identify depressive symptoms. ${ }^{21}$ It is composed of 30 yes/no questions. Answers indicating depression are assigned 1 point and added to yield a total score. The cutoff point to detect depressive symptoms is 10. This assessment was conducted immediately after cognitive assessments.

\section{Physical assessments}

Physical fitness was assessed using three tests from the Senior Fitness Test (SFT) protocol. ${ }^{22}$ Upper and lower limb strength were measured using the arm curl and the sit-to-stand test, respectively. Aerobic capacity was evaluated using the 2-minute step test. These tests are scored according to the age of the participant. The SFT classifies the physical capabilities of older adults at risk of functional loss. For instance, if a woman with 60-64 years perform 11 repetitions in the arm curl, she is at functional risk in the upper limb strength because the cutoff point is at least 12 repetitions. Cutoff points vary according to age and sex, indicating functional limitation to perform a specific task.

Physical assessments were conducted in a single day in the afternoon, 48 hours apart from cognitive assessments.

\section{Statistical analyses}

Descriptive data are shown as mean (standard deviation) and median (minimum and maximum), according to data distribution. Independent T or Mann-Whitney $U$ tests were used to compare cognitive functions, physical capabilities, and depressive symptoms between men and women. As these outcomes were not different $(p>0.05)$, except for lower limb strength, data were pooled, and the sample analyzed as a whole. Variables meeting parametric 
assumptions (global cognition, verbal fluency, short-term memory, upper limb strength, and aerobic capability) were examined using linear regression. To test the influence of physical capabilities on cognition, upper limb strength and aerobic capacity were inserted as independent variables in the linear regression (enter model), while the aforementioned cognitive functions were used as dependent variables.

Logistic regression was performed to calculate the odds ratio (OR) between physical capabilities and cognitive functions, physical capabilities, and depressive symptoms. Missing data were excluded from pairwise analyses. All statistical procedures were performed in JASP 0.13.1. Significance was accepted at the 0.05 alpha level.

\section{Ethics statement}

The study was approved by the Universidade Estadual de Montes Claros ethics committee (protocol 2.398.863/ 2017). All participants voluntarily provided written informed consent to take part in the study.

\section{Results}

The sample was $64 \%$ female. Participants' demographic characteristics are displayed in Table 1. None of the demographic variables was different between males and females $(p>0.05)$. Linear regression showed a relationship between aerobic capacity, upper limb strength, and cognition. However, when age was inserted as a confounding variable, only aerobic capacity predicted $32 \%$ of the variance in global cognition score (adjusted $R^{2}=0.32, B=0.23, t=1.8, p<0.01$ ) and $25 \%$ of the variance in semantic fluency/executive function (verbal fluency) (adjusted $\mathrm{R}^{2}=0.25, \mathrm{~B}=0.44, \mathrm{t}=3.5, \mathrm{p}<0.01$ ). Physical capabilities were not related to short-term memory $(p>0.05)$.

Logistic regression indicated poor cardiorespiratory fitness was associated with semantic fluency/executive function and depressive symptoms. Full data are provided in Table 2.

\section{Discussion}

This study analyzed the associations among physical fitness, cognitive function, and depressive symptoms in a sample of institutionalized older adults. Those with better strength and aerobic capacity were found to exhibit better semantic fluency/executive function. Conversely, older individuals with poor aerobic capacity were most likely to exhibit current depressive symptoms.

Brain connectivity and mental health have been associated with cardiorespiratory fitness. ${ }^{23,24}$ Recently, Plácido et al. $^{10}$ found a strong association among cardiorespiratory fitness measured through the 2-minute step test, Alzheimer's disease (AD), and mild cognitive impairment $(\mathrm{MCl})$. The authors showed that people at risk of functional loss were most likely to be diagnosed with mild $(O R=10.7 ; p=0.001)$ to moderate $(O R=14.7$; $p=0.002) A D$, whereas individuals with low aerobic fitness were 4.5 times more likely to present with $\mathrm{MCl}(p=$ $0.05)$. These findings are in line with ours, showing that poor aerobic capacity in institutionalized older adults is associated with cognitive impairments. The present study extends this literature further by demonstrating that specific cognitive functions, such as semantic fluency/ executive function, are associated with strength and aerobic capacity in institutionalized elderly patients. Those at risk of functional loss were more likely to present a risk up to $38 \%$ of decreased semantic fluency/executive function. In addition, institutionalized older adults with decreased aerobic fitness were more likely to be diagnosed with depressive symptoms $(\mathrm{OR}=1.06,95 \%$ confidence interval $=1.02-1.10, p=0.01)$. Recent literature shows that executive function (e.g., inhibitory control) is acutely improved in individuals who perform aerobic exercise. ${ }^{25}$ In a chronic condition where the individual performs regular exercise, cardiorespiratory fitness is enhanced, which could affect hippocampal connectivity with other

Table 1 Demographic and clinical characteristics of the sample and independent statistical comparison between males and females

\begin{tabular}{lccc}
\hline Characteristic & Males $(\mathrm{n}=27)$ & Females $(\mathrm{n}=49)$ & Total $(\mathrm{n}=76)$ \\
\hline Age (years) & $79 \pm 8$ & $83 \pm 8$ & $82 \pm 8$ \\
Weight $(\mathrm{kg})$ & $76 \pm 14$ & $68 \pm 2$ & $68 \pm 6$ \\
Height (m) & $1.66 \pm 0.06$ & $1.52 \pm 0.06$ & $1.55 \pm 0.08$ \\
Institutionalization (months)* & $15.0(1-156)$ & $24.0(1-96)$ & $1(0-4)$ \\
Chronic diseases (n)* & $2(1-3)$ & $5(0-19)$ & $1(1-156)$ \\
Medications (n)* & $7(1-14)$ & $18 \pm 6$ & $6(0-19)$ \\
MMSE (score) & $19 \pm 7$ & $9 \pm 5$ & $19 \pm 7$ \\
VF (score) & $10 \pm 5$ & $5 \pm 2$ & $9 \pm 5$ \\
DSF (score) & $6 \pm 3$ & $2(0-7)$ & $5 \pm 2$ \\
DSB (score) & $2(0-7)$ & $12 \pm 7$ & $2(0-7)$ \\
GDS (score) & $9 \pm 5$ & $6 \pm 2$ & $11 \pm 7$ \\
Sit-to-stand (repetitions) & $7 \pm 3$ & $33 \pm 17$ & $7 \pm 3$ \\
2-minute step test (repetitions) & $33 \pm 15$ & $8 \pm 4$ & $33 \pm 16$ \\
Arm curl (repetitions) & $11 \pm 4$ & $9 \pm 4$ \\
\hline
\end{tabular}

Data presented as mean \pm standard deviation, unless otherwise specified.

$\mathrm{DSF}$ and DSB = Digit Span forward and backward; GDS = Geriatric Depression Scale; MMSE = Mini-Mental of State Examination; VF = verbal fluency.

* Outcomes described as median (minimum and maximum).

$p<0.05$ between males and females. 
Table 2 Logistic regression associating physical and mental assessments

\begin{tabular}{|c|c|c|c|}
\hline Physical and mental assessments & Odds ratio & $95 \% \mathrm{Cl}$ & $p$-value \\
\hline \multicolumn{4}{|l|}{ Sit-to-stand } \\
\hline Verbal fluency & 1.38 & $1.02-1.85$ & $0.03 *$ \\
\hline Digit Span backward & 1.07 & $0.81-1.41$ & $0.61 *$ \\
\hline Digit Span forward & 1.10 & $0.85-1.42$ & $0.44 *$ \\
\hline Geriatric Depression Scale & 0.87 & $1.07-5.22$ & $0.29^{\dagger}$ \\
\hline \multicolumn{4}{|l|}{ 2-minute step test } \\
\hline Verbal fluency & 1.07 & $1.01-1.14$ & $0.01 *$ \\
\hline Digit Span backward & 1.03 & $0.98-1.09$ & $0.19 *$ \\
\hline Digit Span forward & 1.01 & 0.96-1.05 & $0.59 *$ \\
\hline Geriatric Depression Scale & 1.06 & $1.02-1.10$ & $0.01^{\dagger}$ \\
\hline \multicolumn{4}{|l|}{ Arm curl } \\
\hline Verbal fluency & 1.26 & $1.05-1.52$ & $0.01 *$ \\
\hline Digit Span backward & 1.15 & $0.96-1.38$ & $0.12 *$ \\
\hline Digit Span forward & 1.09 & $0.93-1.28$ & $0.26 *$ \\
\hline Geriatric Depression Scale & 0.90 & $0.78-1.04$ & $0.17^{\dagger}$ \\
\hline
\end{tabular}

Bold types denote significance.

$95 \% \mathrm{Cl}=95 \%$ confidence interval.

* Adjusted by age, gender, long-term care facility, and depressive symptoms.

${ }^{\dagger}$ Adjusted by age, gender, and long-term care facility.

brain regions, such as the medial prefrontal cortex and default mode network (DMN), ${ }^{23}$ explaining the link between physical capacity and improved cognition and depression. The hippocampus and DMN are closely associated to cognition and depression, ${ }^{23,26}$ reinforcing the perspective of physical fitness improving mental health. This is relevant because persons living in LTCFs have poor physical fitness and high physical dependence, which could be associated to poor mental health. ${ }^{7,27}$

While the neurobiological mechanisms underlying physical fitness and cognitive function have not been fully elucidated, a number of studies have shown that persons who are more physically active exhibit better mental health. ${ }^{24,28}$ Many neurobiological processes related to physical fitness, such as improved cerebral blood flow, increased trophic factors, neurotransmitter synthesis, and synaptic branch enhancement, could be associated with brain integrity. ${ }^{29,30}$ Recently, Kronman et al. ${ }^{23}$ found a relationship between cardiorespiratory fitness and improved hippocampus and prefrontal cortex connectivity. The authors showed that people with high levels of cardiorespiratory fitness had more effective connections from the hippocampus to the ventromedial prefrontal cortex, posterior cingulate cortex, lateral temporal cortex, and dorsomedial prefrontal cortex. Interestingly, these brain regions are directly related to episodic memory, short-term and working memory, executive functions, and emotional processing, ${ }^{8,31,32}$ which provides a possible mechanism underlying our findings. Since institutionalized older adults have poor physical fitness, impaired semantic fluency/executive function, and often experience depressive symptoms, they are likely to present with brain atrophy and weak neuronal connections. However, the current study was cross-sectional in nature; longitudinal studies are needed to examine changes in brain structure over time.

These findings may be used to encourage health professionals and LTCF managers to offer physical activities in these institutions, since physical capabilities are directly associated with mental health. Physical activity and supervised exercise have been strongly recommended by the World Health Organization to prevent cognitive decline and reduce the risk of dementia. ${ }^{9}$ Moreover, a position statement published by the Task-force Group suggests physical activity and exercise to improve the health of people living in LTCFs. ${ }^{7}$

The current study has a few limitations. First, the crosssectional design does not allow us to establish causal relationships, although many studies in the literature support a causal link between physical fitness and mood/ cognitive outcomes. Second, data with non-normal distribution were not inserted in the linear regression model. For instance, we could verify whether depression influences cognition and physical capabilities, but depressive symptoms did not meet parametric assumptions. Third, participants with probable dementia (uncertain diagnosis) were retained in the sample, which increased the variability of the data. Finally, full data on some variables, such as years of education, institutionalization period, diagnosis of dementia, and type of medications, were not available from all LTCFs or were missing from our records, and therefore were not inserted as covariates in our analysis. Conducting research in LTCFs is not easy, especially in Brazil, since the majority of these institutions are philanthropic in nature, are not usually supported by the government, and have limited resources (including limited access to specialized health professionals).

In conclusion, physical fitness is associated with cognition and depressive symptoms in institutionalized older adults. In these individuals, poor strength and aerobic fitness increase the odds of presenting with impaired semantic fluency/executive function, which may be associated with prodromal dementia. In addition, poor aerobic fitness was slightly associated with depressive symptoms, showing that sedentary institutionalized older adults could be at particular risk for depression. 


\section{Acknowledgements}

This study was supported by Coordenação de Aperfeiçoamento de Pessoal de Nível Superior (CAPES), Conselho Nacional de Desenvolvimento Científico e Tecnológico (CNPq), and Fundação de Amparo à Pesquisa de Minas Gerais (FAPEMIG). JL is supported by Fundação Carlos Chagas de Amparo à Pesquisa do Estado do Rio de Janeiro (E-26/202.973/2017).

The authors thank Andrea Camaz Deslandes, who contributed important details to Table 1 of the manuscript. We would also like to thank Amber Rochette and the Research and Editing Consulting Program of the International Neuropsychological Society for editorial assistance.

\section{Disclosure}

The authors report no conflicts of interest.

\section{References}

1 World Health Organization (WHO). Global health and aging [Internet]. 2015 [cited 2021 Feb 1]. www.who.int/ageing/publications/global_ health.pdf

2 Hirani V, Blyth F, Naganathan V, Le Couteur DG, Seibel MJ, Waite LM, et al. Sarcopenia is associated with incident disability, institutionalization, and mortality in community-dwelling older men: the concord health and ageing in men project. J Am Med Dir Assoc. 2015;16:607-13.

3 Harmand M, Meillon C, Rullier L, Avila-Funes JA, Bergua V, Dartigues JF, et al. Cognitive decline after entering a nursing home: a 22-year follow-up study of institutionalized and noninstitutionalized elderly people. J Am Med Dir Assoc. 2014;15:504-8.

4 Jerez-Roig J, Ferreira LM, de Araújo JR, Lima KC. Functional decline in nursing home residents: a prognostic study. PLoS One. 2017;12: e0177353.

5 Alves BB, Barbosa EO, Pimentel DM, Carneiro LS, Rodrigues AC, Deslandes AC, et al. Comparison of cognitive functions among frail and prefrail older adults: a clinical perspective. Int Psychogeriatr. 2019;31:297-301.

6 Thakur M, Blazer DG. Depression in long-term care. J Am Med Dir Assoc. 2008:9:82-7.

7 Barreto PS, Morley JE, Chodzko-Zajko W, Pitkala KH, WeeningDjiksterhuis $E$, Rodriguez-Mañas $L$, et al. Recommendations on physical activity and exercise for older adults living in long-term care facilities: a taskforce report. J Am Med Dir Assoc. 2016;17:381-92.

8 Wong CN, Chaddock-Heyman L, Voss MW, Burzynska AZ, Basak C, Erickson $\mathrm{KI}$, et al. Brain activation during dual-task processing is associated with cardiorespiratory fitness and performance in older adults. Front Aging Neurosci. 2015;7:154.

9 World Health Organization (WHO). Risk reduction of cognitive decline and dementia. Geneva: WHO; 2019.

10 Plácido J, Ferreira JV, de Oliveira F, Sant'Anna P, Monteiro-Junior RS, Laks J, et al. Association among 2-min step test, functional level and diagnosis of dementia. Dement Neuropsychol. 2019;13:97-103.

11 Dinas PC, Koutedakis Y, Flouris AD. Effects of exercise and physical activity on depression. Ir J Med Sci. 2011;180:319-25.

12 Barcelos-Ferreira R, Nakano EY, Steffens DC, Bottino CM. Quality of life and physical activity associated to lower prevalence of depression in community-dwelling elderly subjects from Sao Paulo. J Affect Disord. 2013;150:616-22.

13 Kojima G. Prevalence of frailty in nursing homes: a systematic review and meta-analysis. J Am Med Dir Assoc. 2015;16:940-5.
14 Crocker T, Young J, Forster A, Brown L, Ozer S, Greenwood DC The effect of physical rehabilitation on activities of daily living in older residents of long-term care facilities: systematic review with meta-analysis. Age Ageing. 2013;42:682-8.

15 Pereira C, Rosado H, Cruz-Ferreira A, Marmeleira J. Effects of a 10-week multimodal exercise program on physical and cognitive function of nursing home residents: a psychomotor intervention pilot study. Aging Clin Exp Res. 2018;30:471-9.

16 Bertolucci PH, Brucki SM, Campacci SR, Juliano Y. [The Mini-Mental State Examination in a general population: impact of educational status]. Arq Neuropsiquiatr. 1994;52:1-7.

17 Schroeder RW, Twumasi-Ankrah P, Baade LE, Marshall PS. Reliable digit span: a systematic review and cross-validation study. Assessment. 2012;19:21-30.

18 Figueiredo VL, do Nascimento E. Desempenhos nas duas tarefas do subteste dígitos do WISC-III e do WAIS-III. Psic Teor Pesq. 2007;23:313-8.

19 Brucki SM, Malheiros SM, Okamoto IH, Bertolucci PH. [Normative data on the verbal fluency test in the animal category in our milieu]. Arq Neuropsiquiatr. 1997;55:56-61.

20 Passos VM, Giatti L, Barreto SM, Figueiredo RC, Caramelli P, Benseñor I, et al. Verbal fluency tests reliability in a Brazilian multicentric study, ELSA-Brasil. Arq Neuropsiquiatr. 2011;69:814-6.

21 Castelo MS, Coelho-Filho JM, Carvalho AF, Lima JW, Noleto JC Ribeiro KG, et al. Validity of the Brazilian version of the Geriatric Depression Scale (GDS) among primary care patients. Int Psychogeriatr. 2010;22:109-13.

22 Rikli RE, Jones CJ. Functional fitness normative scores for communityresiding older adults, ages 60-94. J Aging Phys Act. 1999;7:162-81.

23 Kronman CA, Kern KL, Nauer RK, Dunne MF, Storer TW, Schon K. Cardiorespiratory fitness predicts effective connectivity between the hippocampus and default mode network nodes in young adults. Hippocampus. 2020;30:526-41.

24 Kandola A, Ashdown-Franks G, Stubbs B, Osborn DP, Hayes JF. The association between cardiorespiratory fitness and the incidence of common mental health disorders: a systematic review and metaanalysis. J Affect Disord. 2019;257:748-57.

25 Formenti D, Cavaggioni L, Duca M, Trecroci A, Rapelli M, Alberti G, et al. Acute effect of exercise on cognitive performance in middleaged adults: aerobic versus balance. J Phys Act Health. 2020 Jul 23; 1-8. doi: 10.1123/jpah.2020-0005. Online ahead of print.

26 Wise T, Marwood L, Perkins AM, Herane-Vives A, Joules R, Lythgoe DJ, et al. Instability of default mode network connectivity in major depression: a two-sample confirmation study. Transl Psychiatry. 2017;7:e1105.

27 Shakeel S, Newhouse I, Malik A, Heckman G. Identifying feasible physical activity programs for long-term care homes in the Ontario context. Can Geriatr J. 2015;18:73-104.

28 Monteiro-Junior RS, Rodrigues VD, Campos C, Paes F, MurilloRodriguez E, Maranhão-Neto GA, et al. The role of physical activity on mood state and functional skills of elderly women. Clin Pract Epidemiol Ment Health. 2017;13:125-33.

29 Deslandes A, Moraes H, Ferreira C, Veiga H, Silveira H, Mouta R, et al. Exercise and mental health: many reasons to move. Neuropsychobiology. 2009;59:191-8.

30 Portugal EM, Cevada T, Monteiro-Junior RS, Guimarães TT, Rubini EC, Lattari E, et al. Neuroscience of exercise: from neurobiology mechanisms to mental health. Neuropsychobiology. 2013;68: 1-14.

31 Monteiro-Junior RS, Vaghetti CA, Nascimento OJ, Laks J, Deslandes AC. Exergames: neuroplastic hypothesis about cognitive improvement and biological effects on physical function of institutionalized older persons. Neural Regen Res. 2016;11:201-4.

32 Santos MA, Bezerra LS, Carvalho AR, Brainer-Lima AM. Global hippocampal atrophy in major depressive disorder: a meta-analysis of magnetic resonance imaging studies. Trends Psychiatry Psychother. 2018;40:369-78. 